\title{
The Dumping Syndrome
}

The English name of "Dumping Syndrome" is not a very descriptive one and may perhaps in the future be discarded. It covers however that group of symptoms which occur after partial gastrectomy , characterized by attacks of weakness, nausea, fainting, and at times epigastric discomfort. It can assume quite severe proportions and was recently described in most illuminating details in the Lancet by a medical sufferer, aged 62. The attacks usually begin when patients first start a full diet and a normal life again after operation. They tend to behave in exactly similar fashion on every occasion they occur, and may persist for years, though in most cases they subside gradually within one or two years of operation. The incidence of this syndrome in gastrectomized patients lies between 5 and 15 per cent, and is certainly highest in the more neurotic individuals. Symptoms almost always occur very soon after a meal, and exertion certainly seems in some cases to bring them on severely.

Earlier views that this syndrome was due to either hypogly-caemia or to hyperglycaemia may be quite discarded-and most authors now regard it as being related in some way to jejunal distension. This is indeed the reason for the name of dumping syndrome which suggests that the post-gastrectomy stomach, emptying itself too rapidly into the anastomotic loop of intestine, causes a sudden distension of this loop with a reflex possibly upon the splanchi-nic vessels. Alvarez has recently commented upon this syndrome and rightly pointed out that it can occur in precisely similar form in certain individuals who have had neither a gastro-enterostomy nor a partial gastrectomy. Such individuals are, he states, hypersensitive over-reactive persons, and may get the syndrome both after eating and also after a bowel action; in either case he regards it merely as a manifestation of an exaggerated reflex. Too rapid filling of the jejunum, or an unduly sensitive nervous system, perhaps with an allergic background, are the main factors involved. If this view of a mechanical distension of the jejunum as the main stimulus for the dumping syndrome is correct, it is rational in treatment to advise slow-eating with small meals, to avoid purely fluid feeds (which pass 